\title{
ZUSAMMENFASSUNGEN
}

\section{Michael Zürn}

Perspektiven des demokratischen Regierens und die Rolle der Politikwissenschaft im 21. Jahrhundert

Die Kernthese des Beitrags lautet: Die originär demokratischen Quellen politischer Legitimität scheinen an Bedeutung zu verlieren. Die Krise der parlamentarischen Demokratie im Westen, der weltpolitische und ökonomische Aufstieg von autoritär verfassten Mächten, die Entstehung politischer Autorität jenseits des Nationalstaates sowie die damit verbundene Entstehung konfligierender Rechtsordnungen verweisen allesamt darauf, dass grundlegende gesellschaftliche und politische Entwicklungen zu einer Verschiebung innerhalb des Sets von etablierten Legitimationsfiguren führen. Originär demokratische Quellen politischer Herrschaft wie Partizipation und öffentliche Deliberation geraten im Zuge der gegenwärtigen Veränderungen der politischen Institutionenlandschaft gegenüber solchen Legitimationsquellen wie Recht, Expertise, Rechenschaftspflichtigkeit und der Steigerung des kollektiven Selbstwertgefühls ins Hintertreffen. Bemerkenswerterweise finden diese Verschiebungen zumeist ein hohes Maß an gesellschaftlicher Akzeptanz. Die genannten Entwicklungen führen somit zu einer Relativierung des demokratischen Prinzips in der Auseinandersetzung über die gute politische Ordnung. Insofern rücken die Frage nach der Begründung von Herrschaft und damit auch die Demokratiefrage wieder in den Mittelpunkt politischer Auseinandersetzungen in der Welt. Das hat weitreichende Implikationen für die Politikwissenschaft als Disziplin - die in Deutschland ja als Demokratiewissenschaft ins Leben gerufen wurde.

\section{Sonja Buckel}

\section{Staatsprojekt Europa}

Der Text untersucht diskursanalytisch die 1998 begonnene und inzwischen stabilisierte Rechtsprechungslinie des Europäischen Gerichtshofes zu den sozialen Rechten Nichterwerbstätiger. Ziel ist es zu zeigen, wie sich gesellschaftliche Auseinandersetzungen in der Arena des Europarechts in rechtliche Auseinandersetzungen übersetzen. Dabei wird die These vertreten, dass der Gerichtshof in der Krise der neoliberalen Hegemonie die unterschiedlichen Kräfte in ein neues hegemoniales juridisches Projekt einbindet, welches eine europäisch-soziale Union anvisiert und einen ersten Hinweis auf ein mögliches ,Staatsprojekt Europa‘ liefert.

\section{Stefan Handke}

\section{Die Angst des Ministeriums vor der Aufsicht - Das Bundesfinanzministerium in der deut- schen Finanzmarktpolitik}

Die Finanzmarktkrise hat gezeigt, dass das Bundesministeriums der Finanzen (BMF) seine Aufgaben in der Finanzmarktpolitik, zu denen vor allem die Schaffung eines Regulierungsrahmens und die Formulierung von Aufsichtsgesetzen gehören, nicht ohne die Unterstüt- 
zung externer Auftragnehmer oder zumindest nachgeordneter Behörden erfüllen kann. Hinsichtlich der Regulierung des Finanzsektors und der Aufsicht über Finanzunternehmen ist das BMF aufgrund einer mangelnden Ressourcenausstattung auf die Bundesanstalt für Finanzdienstleistungsaufsicht (BaFin) und sogar auf Rechtsanwaltskanzleien angewiesen. Daraus ergeben sich zum einen langfristig Probleme für die Politikgestaltungsfähigkeit des Ministeriums in diesem Politikfeld. Zum anderen besteht eine Abhängigkeit des Ministeriums von seiner nachgeordneten Behörde, die es trotz der formal vorhandenen Weisungskompetenz nicht mehr ausreichend zu steuern vermag. Die Handlungsfähigkeit des BMF in der Finanzmarktaufsicht und seine Steuerungskapazität gegenüber der Finanzaufsichtsbehörde sind in einer Weise geschwächt, dass das Ministerium in einer erneut auftretenden Krisensituation nicht zu einer besseren Performanz in der Lage wäre. Bis Ende 2010 diskutierte Reformvorschläge der Finanzaufsicht in Deutschland blendeten dieses Problem jedoch aus und konzentrierten sich ausschließlich auf die BaFin und die Deutsche Bundesbank.

\section{Stefan Schukraft}

Fraktionsgeschlossenheit auf Landesebene im Mehrebenen-Kontext - Der Einfluss des Föderalismus auf den Grad geschlossenen Abstimmungsverhaltens von Fraktionen in den deutschen Landesparlamenten

Der vorliegende Artikel diskutiert einen möglichen Einfluss des Föderalismus auf den Grad geschlossenen Abstimmungsverhaltens von Fraktionen auf Landesebene. Als Antriebsmoment eines solchen Einflusses wird die ebenenübergreifende Konstellation der Regierungskoalitionen herausgearbeitet, die ihren Einfluss über drei verschiedene Wirkungsmechanismen entfaltet. Dabei wird erwartet, dass zunehmende Kongruenz der Koalitionspartner zwischen den beiden gouvernementalen Ebenen dem Grad geschlossenen Abstimmungsverhaltens auf der Landesebene zuträglich ist. Eine empirische Überprüfung kann dies für Abstimmungen, bei denen dissentierendes Abstimmungsverhalten auftritt, bestätigen.

\section{Christian Grobe}

Die Sozialisierung des Homo oeconomicus - Zur Neudefinition rationalen Handelns durch die experimentelle Methode

In den meisten politikwissenschaftlichen Anwendungen von rational choice wird rationales Handeln bis heute als Ergebnis einer konsequenten Maximierung der eigenen materiellen Interessen verstanden. Dabei haben experimentelle Ökonomen über die letzten Jahre die theoretischen Grundlagen des rationalistischen Forschungsprogramms umgewertet und dabei den Homo oeconomicus ,sozialisiert'. In diesem Beitrag soll dieser bedeutende Evolutionsprozess nachgezeichnet werden, um ihn einer breiten politikwissenschaftlichen Leserschaft zugänglich zu machen. Denn dieser Prozess hat einerseits das Potenzial, die disziplininterne Debatte um rational choice in ein neues Licht zu rücken und illustriert andererseits den Mehrwert experimenteller Methoden in der sozialwissenschaftlichen Forschung. Der Beitrag plädiert daher dafür, den sozialisierten Homo oeconomicus und seinen ,Geburtshelfer' - die experimentelle Methode - in Zukunft stärker in der Politikwissenschaft zu berücksichtigen. Der Lohn für diese Erweiterung wäre eine neue Perspektive auf wesentliche Fragen in allen empirisch orientierten Subdisziplinen des Fachs. 